\title{
Cooling holes with different structures effects on coolant cross flow and heat transfer at the outlet of the combustion chamber
}

Ehsan KIANPOUR, Nor Azwadi Che SIDIK

DOI: 10.30464/jmee.2021.5.2.141

Cite this article as:

Kianpour E., Sidik N.A.C. Cooling holes with different structures effects on coolant cross flow and heat transfer at the outlet of the combustion chamber. Journal of Mechanical and Energy Engineering, Vol. 5(45), No. 2, 2021, pp. 141-148.

\section{VOLUME 5(45) | No. 2 | JUNE 2021}

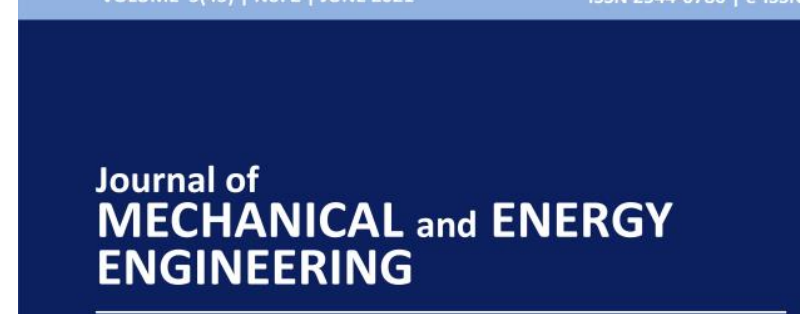

Editor-in-Chief

Waldemar Kuczyński

Editor

Krzysztof Nadolny
Journal of Mechanical and Energy

Engineering

Website: jmee.tu.koszalin.pl

ISSN (Print): 2544-0780

ISSN (Online): 2544-1671

Volume: 5(45)

Number: 2

Year: 2021

Pages: 141-148

Article Info:

Received 10 June 2021

Accepted 25 June 2021

\section{Open Access}

This article is distributed under the terms of the Creative Commons Attribution 4.0 (CC BY 4.0) International License (http://creativecommons.org/licenses/by/4.0/), which permits unrestricted use, distribution, and reproduction in any medium, provided you give appropriate credit to the original author(s) and the source, provide a link to the Creative Commons license, and indicate if changes were made. 


\title{
COOLING HOLES WITH DIFFERENT STRUCTURES EFFECTS ON COOLANT CROSS FLOW AND HEAT TRANSFER AT THE OUTLET OF THE COMBUSTION CHAMBER
}

\author{
Ehsan KIANPOUR ${ }^{1 *}$, Nor Azwadi Che SIDIK ${ }^{2}$ \\ ${ }^{1 *}$ Department of Mechanical Engineering, Najafabad Branch,Islamic Azad University, Najafabad, Iran \\ ekianpour@pmc.iaun.ac.ir \\ ${ }^{2}$ Department of Thermo-fluid, Faculty of Mechanical Engineering, University Technology of Malaysia, \\ Skudai, Johor, Malaysia
}

(Received 10 June 2021, Accepted 25 June 2021)

\begin{abstract}
The major effects of cylindrical and trenched cooling holes with angles of $\alpha=30^{\circ}$, $\beta=0^{\circ}, \alpha=40^{\circ}, \beta=0^{\circ}$ and $\alpha=50^{\circ}, \beta=0^{\circ}$ at $\mathrm{BR}=3.18$ on the film cooling effectiveness near the combustor end wall surface is an important subject to study in detail. In the current study, the researchers used a FLUENT package 16/11 to simulate a 3-D model of a Pratt and Whitney gas turbine engine. In this research, an RNG K- $\varepsilon$ turbulence model was used to analyze the flow behavior on the passage ways of internal cooling. In the combustor simulator, the dilution jets and the cooling flow staggered in the streamwise direction and it aligned in the spanwise direction, as well. In comparison with the baseline case of cooling holes, the application of trenched holes near the end wall surface increased the effectiveness of film cooling up to $100 \%$ for different cases.
\end{abstract}

Keywords: gas turbine engine, film-cooling, combustor simulator, trench hole, dilution hole

\section{INTRODUCTION}

Advanced gas turbine industries are aiming for higher engine efficiencies. The Brayton cycle is a key to achieve this purpose. In this cycle, to have a higher gas turbine engine efficiency, the combustor's outlet temperature must increase [1]. But such hot flows cause non-uniformities at the end of the combustor and the inlet of the turbine and damage the critical parts. Film cooling is the most well-known method of preservation. In this technique, a low temperature thin layer attaches on a surface and protects it against hot streams. To obtain a better film cooling performance, it is needed to increase the blowing ratio. The blowing ratio increment has an intense effect on the heat transfer, particularly in the hole region. According to the importance of this study, a broad literature survey was done to obtain fundamental data. Anderson et al. [2] investigated the separate effects of the Mach and Reynolds numbers, and the boundary layer thickness on the effectiveness of the cooling holes shaped. In this study, a parametric study of these effects was performed. This study was performed using cooling hole diameters of $D=4.0 \mathrm{~mm}$ and $9.0 \mathrm{~mm}$, the flow rate Mach number of 0.03 to 0.15 and the Reynolds number of 5.500 to 15.500 . The effect of the boundary layer properties, including the layered and turbulent boundary layer properties as well as different boundary layer thicknesses, was also investigated. The results showed that at high freestream turbulence conditions, the performance increased with an increasing thickness of the approach boundary layer in contrast to the decrease in performance seen previously for cylindrical holes. In line with this study, Abdullah and Funazaki [3] analyzed the effects of hole angle geometries on film cooling. They considered four different rows of inclined holes with angles of 20 degrees and 35 degrees. They made ready the contours which showed the laterally averaged film cooling distribution and cooling performance at $\mathrm{x} / \mathrm{D}$ equal to 3,13, 23 and 33. The results demonstrate that at a higher blowing ratio $(\mathrm{BR}=3.0$ and 4.0$)$, the interaction between the neighboring secondary airs leads to a full coverage film cooling effectiveness downstream of the fourth row, which was confirmed by the temperature field captured at $x / D=33$ and $\mathrm{BR}=4.0$. 
In addition, Nasir et al. [4], Shine et al. [5] and Zhang and Wang. [6] simulated a flat plate with cylindrical cooling holes to study the effects of the injection angle on the effectiveness of film cooling. They highlighted that lower streamwise injection angles perform better to obtain a higher film cooling effectiveness. Tarchi et al. [7] investigated the effects of large dilution holes. These holes were placed within the injecting slot and eruption array. The flat plate cross section duct contained 270 cooling holes located in 29 staggered rows. The holes were $1.65 \mathrm{~mm}$ in diameter and had a length to diameter ratio of 5.5 and a stream-wise angle of 30 degrees. The dilution hole was $18.75 \mathrm{~mm}$ in diameter. It was located at the 14th row of cooling holes. Tarchi et al., Milanes et al. [8], Dai et al. [9] and Li et al. [10] showed that with using a backward step, at downstream the dilution holes the adiabatic film cooling effectiveness reached to $\eta=0.65$. Vakil and Thole [11] presented experimental results of the study of the temperature distribution inside a combustor simulator. In this study, a real large scale of the combustor was modelled. This model contained four different cooling panels with many cooling holes. Two rows of dilution jets could be seen in the second and third cooling panels. The first row had three dilution jets and the second one had two jets. While the first and second panels were flat, two other panels angled with an angle of 15.8 degrees. The results indicated that high temperature gradient was developed upstream of the dilution holes. Kianpour et al. [12] re-simulated the Vakil and Thole's combustor. They offered various geometries of the cooling holes. The temperatures near the wall and among the jets were higher for the baseline cooling whereas the central part of the jets was cooler in trenched cases. By using the control volume technique and the RNG $\mathrm{k}-\varepsilon$ turbulence model, Zhang et al. [13] investigated the flow and heat transfer behavior on a flat plate film cooling from the coneshaped and round-shaped cooling holes. The results showed that at the same blowing ratio, the film cooling effectiveness for the cone-shaped holes is better than for the round-shaped holes. At last, for cone-shaped jets, the jet to the cross flow blowing ratio reaches the optimum condition of 1.0 to yield the best film cooling effectiveness (Gao et al. [14]; Colban et al. [15]). In addition, Cao et al. [16], Saumweber and Schulz [17] and Barigozzi et al. [18] indicated that better thermal protection is attained at higher blowing ratios. Hou et al. [19] studied the film cooling effectiveness with different compound angles and coolant inlet directions at blowing ratio $\mathrm{BR}=1.0$ by Large Eddy Simulation (LES). The compound angle was 0 and 90 degrees, which is commonly used in the real program. The results show that the combined angle of 90 degrees creates an asymmetric downstream vortex and it increases the complexity of the flow characteristics. The trench hole combined angle of $0^{\circ}$ narrows the velocity distribution difference at the trench outlet due to the redistribution of the coolant in the trench. Song et al. [20] studied the effects of three trench height configurations, the distance from the hole exit to the down-stream side wall and the radius of the curvature of the downstream side wall on the film cooling effectiveness of the fan-shaped hole experimentally. The film cooling effectiveness was measured using pressure sensitive paint (PSP) and the experiments were performed with the blowing ratios of 1.0, 1.5, 2.0, and 2.5 and the density ratios of 1.0 and 2.0. The results showed that the cooling effect of the film inside the trench is generally greater for cases with a larger trench height. In agreement with the study background, several authors motivate the Author to conduct this research. The end wall of the combustor can be damaged by the hot gases which flow inside a combustor simulator and increasing the film cooling effectiveness above these surfaces is an important issue which attracts less attention till now. In addition, most of the studies paid attention on the use of trenched holes at the leading edge of the turbine blades, and in most of them the application of these holes at the end wall of the combustor is not considered.

\section{MATERIALS AND METHODS}

In the present study, the combustor simulator was a 3-D representation of a Prat and Whitney gas turbine engine. As seen in Fig. 1, the combustor was a threedimensional container. The width, height and length of this container was $111.8 \mathrm{~cm}, 99.1 \mathrm{~cm}$ and $156.9 \mathrm{~cm}$ respectively. The container converged from $x / L=0.51$ and the contraction angle was 15.8 degrees. The inlet and outlet cross-sectional area of the combustor simulator was $1.11 \mathrm{~m}^{2}$ and $0.62 \mathrm{~m}^{2}$. The test section contained two symmetric surfaces on the top and bottom of the combustor but the fluid only flowed through the bottom passage. The lengths of the panels were $39 \mathrm{~cm}, 41 \mathrm{~cm}, 37 \mathrm{~cm}$ and $43 \mathrm{~cm}$ respectively. In addition, the first two panels were flat and possessed a constant sectional area. However, the last two panels were inclined at the contraction angle. The panels were $1.27 \mathrm{~cm}$ thick, and due to the low thermal conductivity $(\mathrm{k}=0.037 \mathrm{~W} / \mathrm{mk})$, adiabatic surface temperature measurements were possible. Two different dilution rows were considered within the second and third panels of cooling panels. The dilution flow injected into the mainstream flow vertically, while the dilution hole in the third panel was angled at $15.8^{\circ}$ from the vertical axis. The first row of the dilution jets included three holes and it was placed at $0.67 \mathrm{~m}$ downstream of the combustor simulator inlet. These holes were $8.5 \mathrm{~cm}$ in diameter. The second row contained two dilution holes and it was located at $0.23 \mathrm{~m}$ downstream of the first row of the dilution holes center. These holes diameter was $12.1 \mathrm{~cm}$. The centerline of the second row was staggered with respect to those of the first row. The 
length of these cooling holes was $2.5 \mathrm{~cm}$ and they were drilled at an angle of $30^{\circ}$ from the horizontal surface. The film-cooling holes were $0.76 \mathrm{~cm}$ in diameter. Except for the baseline case which was introduced, trenched cooling holes with angles of $\alpha=30^{\circ}, \beta=0^{\circ}$, $\alpha=40^{\circ}, \beta=0^{\circ}$ and $\alpha=50^{\circ}, \beta=0^{\circ}$ were considered (Fig. 2).

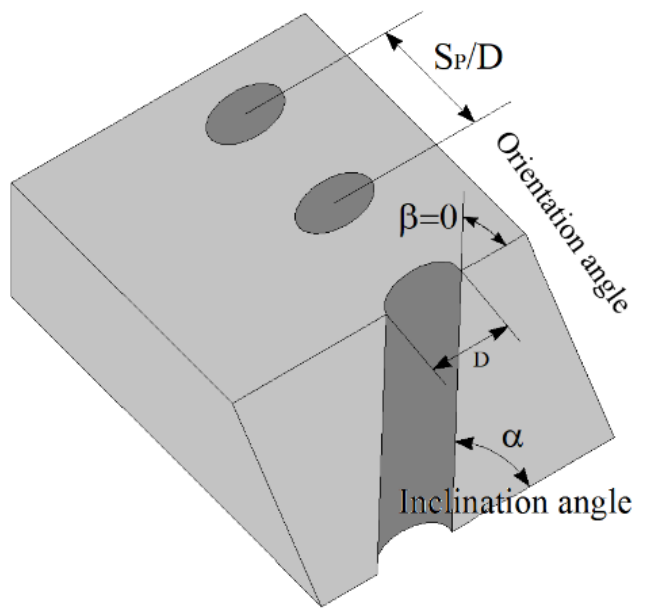

Fig. 1. Schematic view of cylindrical cooling hole

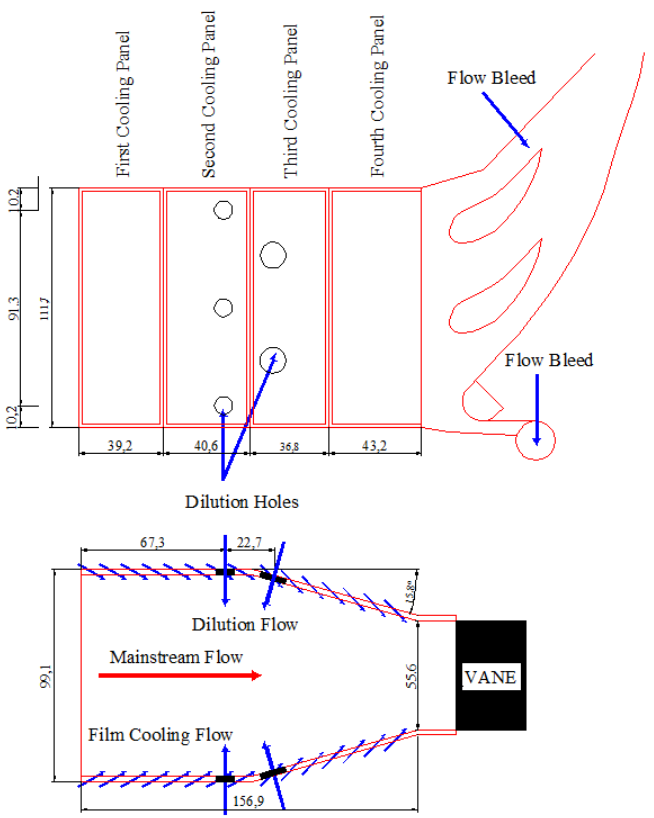

Fig. 2. Schematic view of combustor simulator

Furthermore, coolant blowing ratios were equal to $\mathrm{BR}=3.18$. The cartesian coordinate system $(x, y$ and $z)$ was selected. The temperature of the coolant and dilution jets was equal to $295.5 \mathrm{~K}$. The temperature of the mainstream was 332 K. In Fig. 3, the observation planes which are used to measure the film cooling effectiveness distribution for the baseline case and four different configurations of trenched cooling holes were shown. The observation planes of $0 p, 1 p, 2 p$, and $3 p$ and $1 \mathrm{~s}$ were placed in pitch wise and streamwise directions respectively. Plane $0 p$ was located at $x=35.1 \mathrm{~cm}$. The distribution of the film cooling momentum was computed along this panel. Plane $1 \mathrm{p}$ was located at the trailing edge of the first row of dilution jet. It was used to identify the effects of film cooling and the dilution jets interaction, the horseshoe, half-wake and counter rotating vortexes effects. Plane $2 p$ was placed at the trailing edge of the second row of dilution jets. The interaction between the first and second rows of dilution jets was distinguished by the plane usage. Plane $3 \mathrm{p}$ was located at the combustor simulator exit. It was applied to determine the behavior of the outlet flow and the varying combustor temperature.

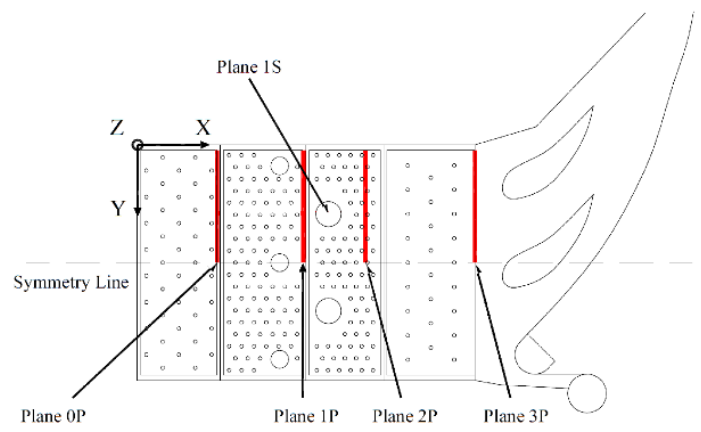

Fig. 3. Location of observation planes

About $8 \times 10^{6}$ tetrahedral meshes were selected (Fig. 4). The meshes were denser around the cooling and dilution holes as well as the wall surfaces.

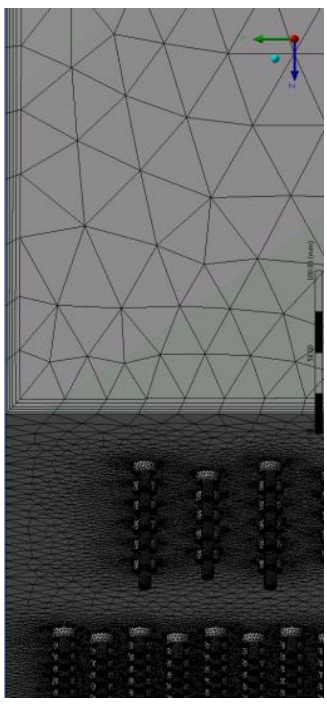

Fig. 4. Meshes of combustor simulator

According to the blowing ratio considered at the inlet of control volume, the boundary condition of the inlet mass flow was considered at the inlet to limit the interaction region between the fluid and combustor 
wall, the slip-less boundary condition and the wall boundary condition were considered. In addition, two different boundary conditions of the uniform flow and the pressure outlet were selected at the inlet and outlet of the combustor respectively. Totally, according to the symmetries of the Pratt and Whitney gas turbine engine combustor, the symmetry boundary condition was used. The numerical method considered a transient and incompressible turbulent flow by means of the $\mathrm{k}-\varepsilon$ turbulent model of the Navier-Stokes equations expressed as follows:

Continuity equation

$$
\frac{\partial}{\partial t}\left(\rho u_{i}\right)+\frac{\partial}{\partial x_{j}}=\left(\rho u_{i} u_{j}\right)=-\frac{\partial \mathrm{P}}{\partial x_{i}}+\frac{\partial \tau_{i j}}{\partial x_{i}}+\rho g_{i}+\vec{F}_{1}
$$

Momentum equation

$$
\frac{\partial \rho}{\partial t}+\frac{\partial \rho}{\partial x} \frac{d x}{d t}+\frac{\partial \rho}{\partial y} \frac{d y}{d t}+\frac{\partial \rho}{\partial z} \frac{d z}{d t}=-\rho(\nabla \cdot V) .
$$

Energy equation

$$
\begin{aligned}
& \frac{\partial}{\partial t}(\rho E)+\frac{\partial}{\partial x_{i}}=\left(u_{i}(\rho E+P)\right)= \\
& \frac{\partial}{\partial x_{i}}\left(K_{e f f} \frac{\partial T}{\partial x_{i}}-\sum_{j} h_{j} J_{j}+u_{i}\left(\tau_{i j}\right)_{e r f}\right)+S_{h}
\end{aligned}
$$

and $\mathrm{k}-\varepsilon$ equation

$$
\begin{gathered}
\frac{\partial}{\partial t}(\rho k)+\frac{\partial}{\partial x_{i}}\left(\rho k u_{i}\right)=\frac{\partial}{\partial x_{i}}\left(\mu+\frac{\mu_{t}}{\sigma k} \frac{\partial k}{\partial x_{j}}\right)+P_{k}-\rho \varepsilon \\
\frac{\partial}{\partial t}(\rho \varepsilon)+\frac{\partial}{\partial x_{i}}\left(\rho \varepsilon u_{i}\right)= \\
\frac{\partial}{\partial x_{j}}\left(\left(\mu+\frac{\mu_{t}}{\sigma \varepsilon}\right) \frac{\partial \varepsilon}{\partial x_{j}}\right)+C_{1 \varepsilon} \frac{\varepsilon}{k} P_{k}-C_{2 \varepsilon}^{*} \rho \frac{\varepsilon^{2}}{k}
\end{gathered}
$$

To investigate the convergence limit, the control volume mass residue was estimated and the maximum value was used. For this research, the criterion of convergence was chosen of $10^{-4}$. The following equation is to determine the effectiveness of filmcooling.

$$
\eta=\frac{T-T_{\infty}}{T_{c}-T_{\infty}}
$$

ere, $\mathrm{T}, \mathrm{T}_{\infty}$ and $\mathrm{T}_{\mathrm{c}}$ is the local temperature, the temperature mainstream and coolant respectively.

\section{FINDINGS AND DISCUSSION}

The findings of the current research were compared with the experimental results collected, which was done by Vakil and Thole [11], and the numerical findings obtained by Stitzel and Thole [21]. The effectiveness of film-cooling was compared in plane $1 p$ and $2 p$ at $y / w=0.4$ (Fig. 5). The deviations between the results of the current research and the benchmarks were computed using the following equation:

$$
\% \text { Diff }=\left(\left(\sum_{i=1}^{n} \frac{x_{i}-x_{i, \text { benchmark }}}{x_{i, \text { benchmark }}}\right) / n\right) \times 100 .
$$

The deviation was equal to $9.76 \%$ and $8.34 \%$ compared to the Vakil and Thole measurements [11] and the Stitzel and Thole [21] estimation for plane 1p and $13.36 \%$ and $11.96 \%$ in comparison with Ref [11] and Ref [21] for plane $2 \mathrm{p}$.
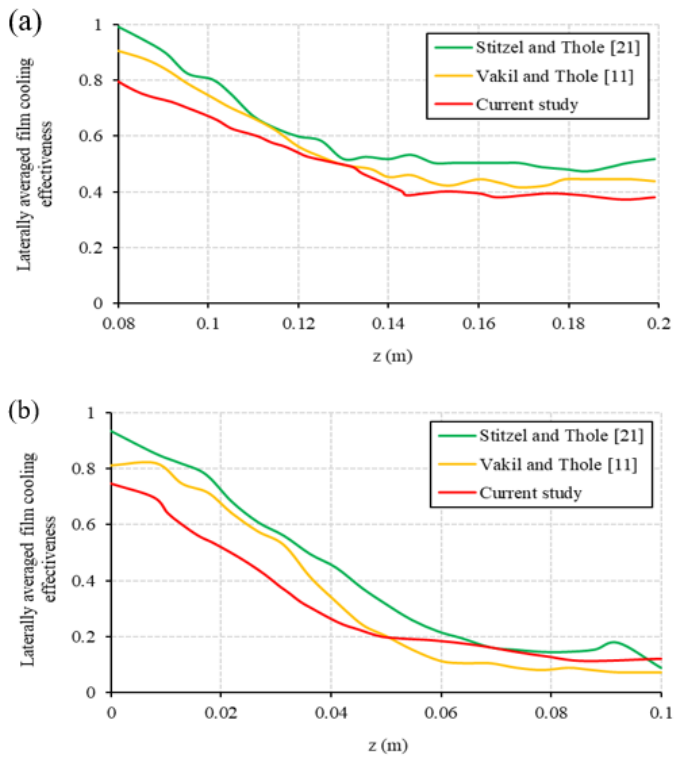

Fig. 5. Film cooling effectiveness comparison of (a) plane $1 \mathrm{p}$ and $(\mathrm{b})$ plane $2 \mathrm{p}$ along $\mathrm{y} / \mathrm{W}=0.4$

Much of the film cooling effectiveness data in this study was collected on the assumption that symmetry could be applied within the combustor simulator. A vertical film cooling effectiveness distribution taken at the intersection of panels $0 p$ which extended over approximately $10 \%$ of the total inlet height at the blowing ratio of 3.18 is shown in Fig. 6. The jets closest to the observation plane had their coolest region near the wall with a secondary cool region directly above it. This secondary cool region was a remnant of the upstream film cooling jet that exited from the aligned cooling hole. The results also showed that the penetration depth for the simulated combustion chamber for the holes with angles $\alpha=30^{\circ}, \beta=0^{\circ}$ and $\alpha=50^{\circ}, \beta=0^{\circ}$ was greater than the baseline model. The film cooling effectiveness in plane 1p was taken directly downstream of the first row of the dilution jets and it is shown in Fig. 7. This particular hole is centrally located right at the mid pitch within the combustor simulator. The results illustrate that the center of the dilution jet is reasonably cantered about the corners of this observation plane as the dilution jet surges up. 
(a)

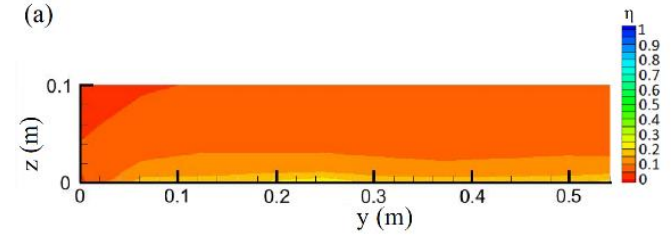

(b)

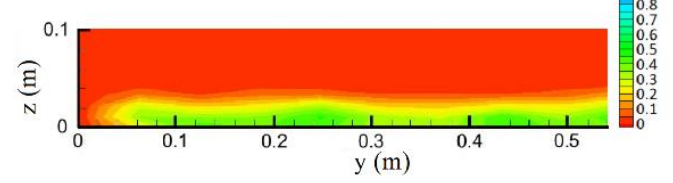

(c)

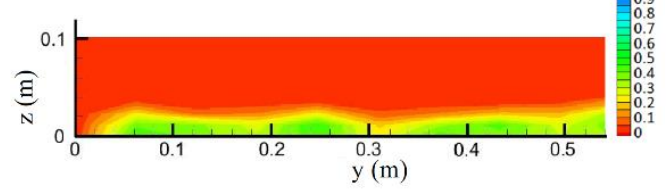

(d)

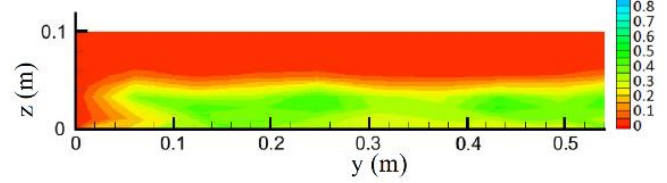

Fig. 6. Film cooling effectiveness of plane $0 \mathrm{p}$ (a) baseline case (b) $\alpha=30^{\circ}, \beta=0^{\circ}$, (c) $\alpha=40^{\circ}, \beta=0^{\circ}$ (d) $\alpha=50^{\circ}, \beta=0^{\circ}$

(a)

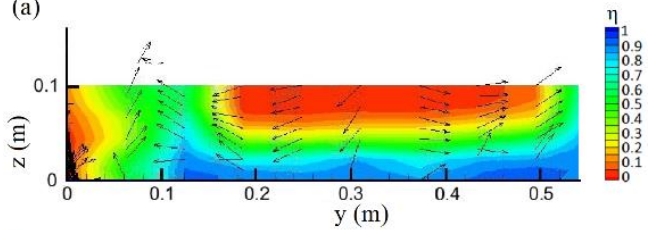

(b)

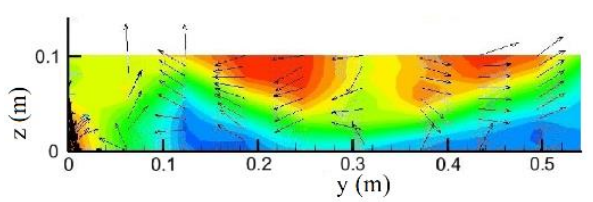

(c)

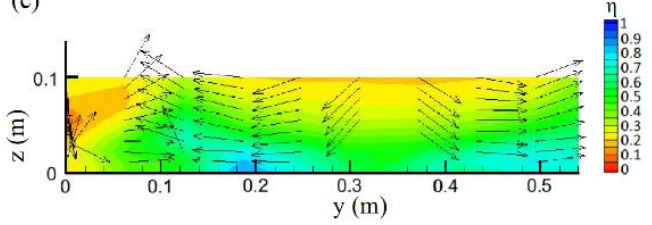

(d)

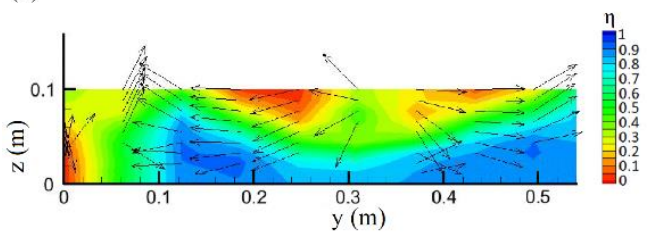

Fig. 7. Film cooling effectiveness for plane $1 \mathrm{p}$ (a) baseline case (b) $\alpha=30^{\circ}, \beta=0^{\circ}$, (c) $\alpha=40^{\circ}, \beta=0^{\circ}$ (d) $\alpha=50^{\circ}, \beta=0^{\circ}$
Also, the results show the effect of increasing the coolant momentum flux relative to the interaction with the mainstream. It is to be noted that when film cooling significantly increased, the dilution jet injection remained the same. This figure also shows slightly higher levels near the wall for the trenched cases relative to the baseline, especially for $\alpha=30^{\circ}, \beta=0^{\circ}$ case. Moreover, the temperature was slightly higher $(0<\eta<0.1)$ for the base line, $\alpha=30^{\circ}, \beta=0^{\circ}$ and $\alpha=$ $50^{\circ}, \beta=0^{\circ}$ cases at the position of $18 \mathrm{~cm}<y<28 \mathrm{~cm}$ and $38 \mathrm{~cm}<y<50 \mathrm{~cm}$. The contours of film cooling effectiveness in plane $2 p$ at $B R=3.18$ located one dilution hole diameter (1D2) downstream of the trailing edge of a two hole dilution were shown in Fig. 8. As it can be seen to the right of the contours, the mushroom shaped temperature profiles got mixed and a warmer region near the mid-span emerged.

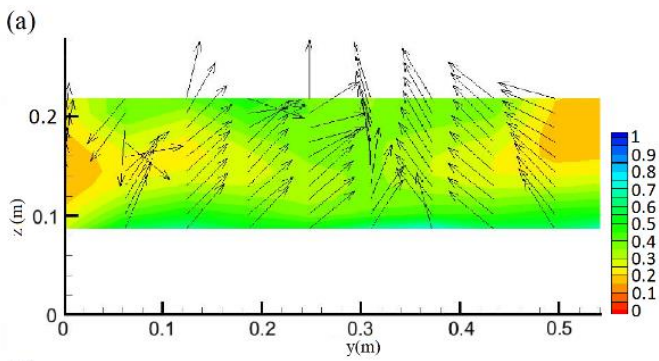

(b)

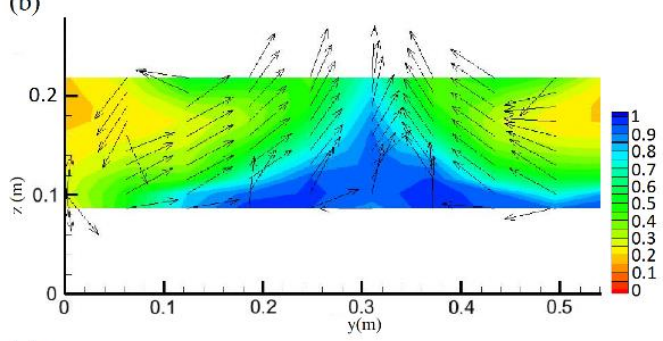

(c)

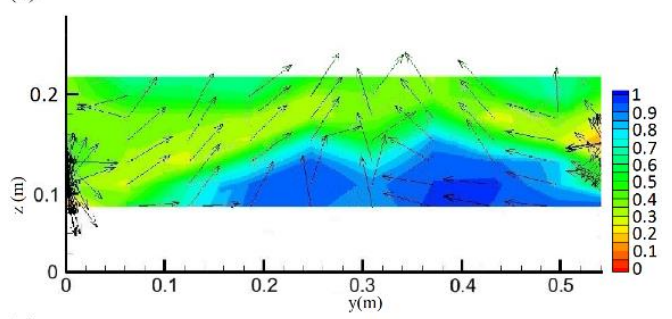

(d)

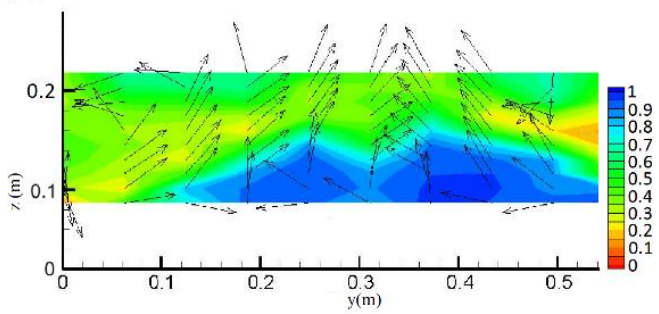

Fig. 8. Film cooling effectiveness in plane $2 \mathrm{p}$ (a) baseline case (b) $\alpha=30^{\circ}, \beta=0^{\circ}$, (c) $\alpha=40^{\circ}, \beta=0^{\circ}$ (d) $\alpha=50^{\circ}, \beta=0^{\circ} 0$ 
There is a probability that the warm region is formed as a result of the flow of an unmixed warmer fluid around the stagnation region caused by the impact of opposing dilution jets in the first row. Fig. 9 shows the film cooling effectiveness distribution for plane $3 p$ and illustrates the behavior of the flow at the end of the combustor simulator where $x / L$ is equal to 1.0. For these cases, the mushroom shaped temperature profiles have mixed together and a warmer region on the corners has emerged.

(a)

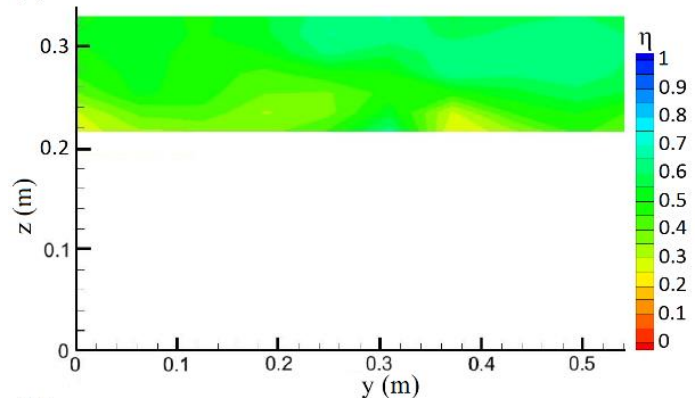

(b)

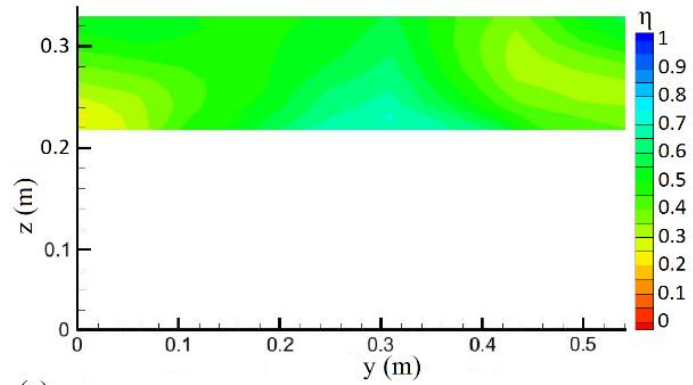

(c)

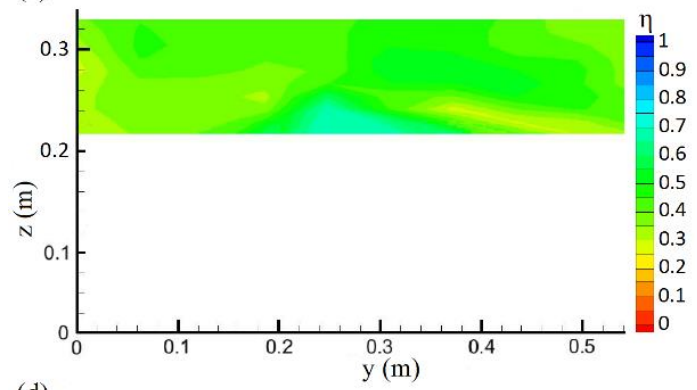

(d)

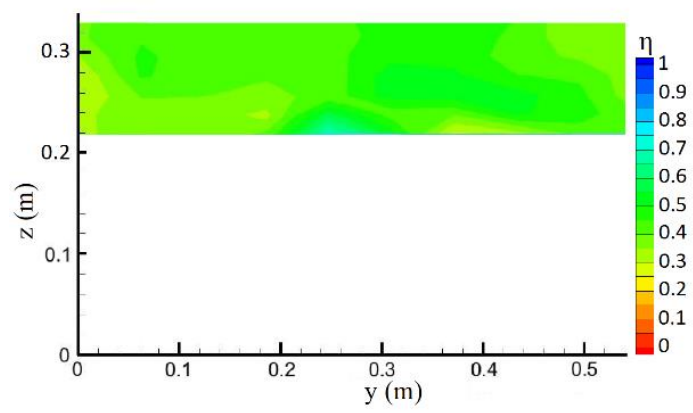

Fig. 9. Film cooling effectiveness in plane $3 p$ (a) baseline case (b) $\alpha=30^{\circ}, \beta=0^{\circ}$, (c) $\alpha=40^{\circ}, \beta=0^{\circ}$ (d) $\alpha=50^{\circ}, \beta=0^{\circ}$
This warmth results from the unmixed warmer flowing around the stagnation region which was created by the dilution jets inter-action in the second row. The velocity vectors show a strong downward and midground current due to contraction in the combustion chamber. A dominant anti-clockwise vortex is clearly shown in this figure. Fig. 10 shows the temperature contour on a transverse plane $1 \mathrm{~s}$.

(a)

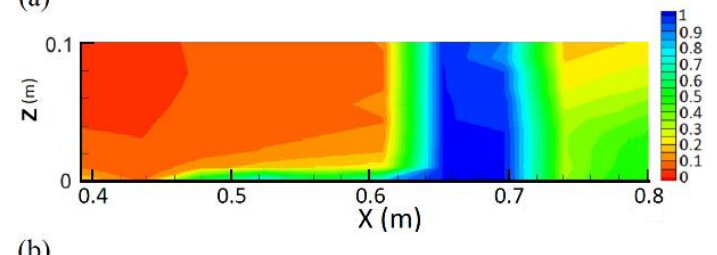

(b)

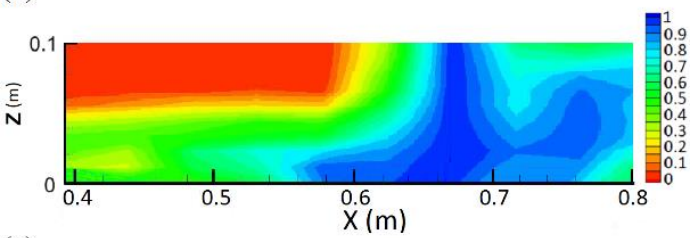

(c)

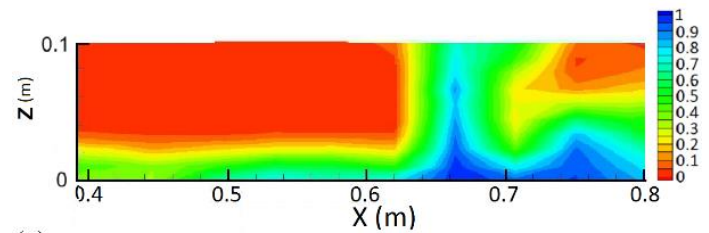

(e)

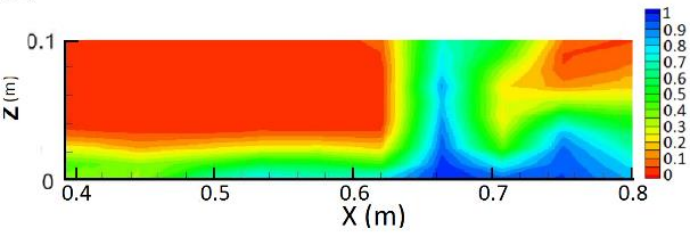

Fig. 10. Film cooling effectiveness for plane $1 \mathrm{~s}$ (a) baseline case (b) $\alpha=30^{\circ}, \beta=0^{\circ}$, (c) $\alpha=40^{\circ}, \beta=0^{\circ}$ (d) $\alpha=50^{\circ}, \beta=0^{\circ}$

The shear forces effects downstream the dilution jet is shown in this figure. As it can be seen, a thinner layer of a low temperature film is formed immediately downstream the dilution jets and it is entrained into the jet. Since the effects of entrainment became weaker further from the dilution jets, the reverse flow occurred and the vectors followed the temperature contours, whereas the vectors thickened under the effects of downstream turbulence growing. For these cases, the mushroom shaped temperature profiles mixed together and a warmer region on the corners emerged. This warmth is the result of the unmixed warmer flow around the stagnation region which was created by the dilution jets inter-action in the second row. In the model $\alpha=30^{\circ}, \beta=0^{\circ}$, the emission of the second-row diluent jet is much better than for the other models.

The changes of the film cooling effectiveness of different configurations for the observation plane of $1 \mathrm{p}$ 
and $2 p$ at the blowing ratio of $B R=3.18$ can be observed in Fig. 11. The results obtained were plotted at the center of span-wise direction and along the vertical axis downstream the edge of the second and third cooling panels with different alignment angles $\alpha=30^{\circ}, \beta=0^{\circ}, \alpha=40^{\circ}, \beta=0^{\circ}$ and $\alpha=50^{\circ}, \beta=$ $0^{\circ}$. This was done to quantitatively analyze the findings associated with the data collected throughout our computational study using the FLUENT software. At this blowing ratio, the cooling holes with an angle of $\alpha=40^{\circ}, \beta=0{ }^{\circ}$ degrees performed the most effectively. This was 35 percent as much the film cooling effectiveness of the cooling holes with the angles of $\alpha=50^{\circ}, \beta=0^{\circ}$.

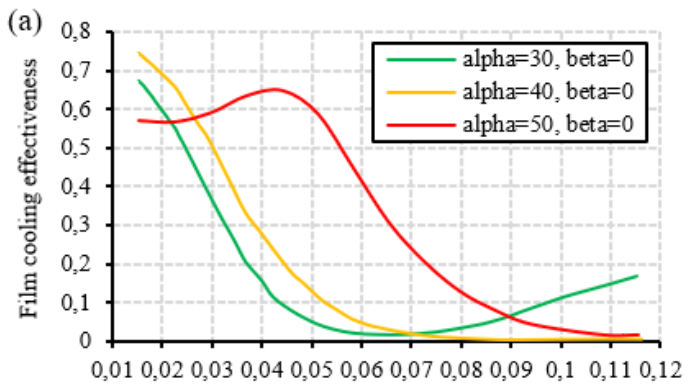

$\mathrm{z}(\mathrm{m})$

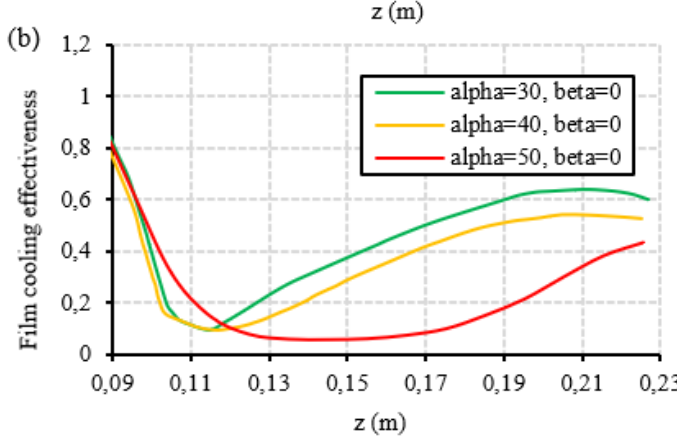

Fig. 11. Effect of different cooling cases in planes $1 p$ and $2 p$ on film cooling effectiveness distribution with blowing ratios of $\mathrm{BR}=3.18$

\section{CONCLUSIONS}

In this research, a numerical study was conducted in order to have a better understanding of the film cooling effectiveness and the effects of cooling holes with different angles of $\alpha=30^{\circ}, \beta=0^{\circ}, \alpha=40^{\circ}$, $\beta=0^{\circ}$ and $\alpha=50^{\circ}, \beta=0^{\circ}$ from the combustor exit of a gas turbine engine. An optimized design of the cooling holes will help to maximize the effectiveness of cooling along the combustor end wall surface and to prevent premature wear in this area. A threedimensional representation of a gas turbine engine was simulated in order to analyze the effects of trenched cooling holes. The combustor simulator combined the effects of two rows of streamwise staggered and spanwise aligned dilution jets. The commercial FLUENT software and RNG $\mathrm{k}-\varepsilon$ turbulence model were employed to run the computations on the thermal fields within a combustor simulator under the blowing ratio of 3.18. Compared to the baseline method, the cooling holes with different angles, the coolant remained closer to the end wall surface and it did not allow main entrainment. It also provided significant lateral spreading and stronger coverage. It appears that cooling holes with different angles of $\alpha=30^{\circ}, \beta=0^{\circ}$, $\alpha=40^{\circ}, \beta=0^{\circ}$ and $\alpha=50^{\circ}, \beta=0^{\circ}$ increase film cooling effectiveness, especially for the case of $\alpha=30^{\circ}, \beta=0^{\circ}$. Film cooling was also explored at the blowing ratio of 3.18 and the observation plane of $0 \mathrm{p}$ and $2 \mathrm{p}$, using cooling holes with the angles of $\alpha=30^{\circ}, \beta=0^{\circ}$. However, for plane $1 \mathrm{p}$, i.e., at the trailing edge of the second cooling panel, the cooling holes with the angles of $\alpha=50^{\circ}, \beta=0^{\circ}$ increased film cooling effectiveness by $75 \%$, which is much higher than for the other configurations.

\section{References}

1. Ai W. R., Laycock G., Rappleye D. S., Fletcher T. H., Bons J. P. (2011). Effect of particle size and trench configuration on deposition from fine coal flyash near film cooling holes. Energy and Fuels, Vol. 25, pp. 1066-1076.

2. Anderson J. B., Wilkes E. K., McClintic J. W., Bogard D. G., (2016), Effects of freestream Mach number, Reynolds number, and boundary layer thickness on film cooling effectiveness of shaped holes. In Turbo Expo: Power for Land, Sea, and Air, Vol. 49804, pp. V05CT19A003).

3. Abdullah K., Funazaki K. I. (2012). Effects of Blowing Ratio on Multiple Shallow Angle Film Cooling Holes. In Applied Mechanics and Materials, Vol. 225, pp. 49-54.

4. Nasir H., Ekkad S., Acharya V. S. (2001). Effect of compound angle injection on flat surface film cooling with large streamwise injection angle. Experimental Thermal and Fluid Science Journal, Vol. 25, No. 1-2, pp. 23-29.

5. Shine S.R., Sunil Kumar S., Suresh B. N. (2013). Internal wall-jet film cooling with compound angle cylindrical holes. Energy Conversion and Management, Vol. 68, pp. 54-62.

6. Zhang C., Wang Z., (2018). Effect of the downstream crescent-shaped block height on the flat-plate film flow and cooling performance. Journal of Applied Mechanics and Technical Physics, Vol. 59, No. 5, pp.951-961.

7. Tarchi L., Facchini B., Maiuolo F., Coutandin D. (2012). Experimental Investigation on the Effects of a Large Recirculating Area on the Performance of an Effusion Cooled Combustor Liner. Journal of Engineering for Gas Turbines and Power, Vol. 134, No. 4, pp. 041505-1-041505-9.

8. Milanes D. W., Kirk D. R., Fidkowski K. J., Waitz I. A. (2006). Gas Turbine Engine Durability Impacts of High Fuel-Air Ratio Combustors: Near Wall Reaction Effects on Film-Cooled Backward-Facing Step Heat Transfer. Journal of Engineering for Gas Turbines and Power, Vol. 128, No. 2, pp. 318-325.

9. Dai H., Zhang J., Ren Y., Liu N., Lin, J., (2021). Effect of cooling hole configurations on combustion and heat transfer in an aero-engine combustor. Applied Thermal Engineering, Vol. 182, pp.115664.

10. Li L., Liu T., Peng X. F. (2005). Flow characteristics in an annular burner with fully film cooling. Applied Thermal Engineering, Vol. 25, No. 17-18, pp. 3013-3024.

11. Vakil S. S., Thole K. A. (2005). Flow and Thermal Field Measurements in a Combustor Simulator Relevant to a Gas Turbine Aero engine. Journal of Engineering for Gas Turbines and Power, Vol. 127, No. 2, pp. 257-267.

12. Kianpour E., Sidik N.A.C., Golshokouh I. (2014). Film cooling effectiveness in a gas turbine engine: a review. Jurnal Teknologi, Vol. 71, No. 2, pp. 25-35. 
13. Zhang X. Z., Hassan I. (2006). Numerical investigation of heat transfer on film cooling with shaped holes. International Journal of Heat and Fluid Flow, Vol. 16, No. 8, pp. 848-869.

14. Gao Z., Narzary D., Han J. C. (2009). Turbine Blade Platform Film Cooling With Typical Stator-Rotor Purge Flow and Discrete-Hole Film Cooling. Journal of Turbomachinery, Vol. 131, No. 4, pp. 041004-1-041004-11.

15. Colban W., Thole K. A., Haendler M. (2008). A comparison of cylindrical and fan-shaped film-cooling holes on a vane end wall at low and high freestream turbulence levels. Journal of Turbomachinery, Vol. 130, No. 3, pp. 031007-1-031007-9.

16. Cao N., Li X., Wu Z., Luo X., (2020). Effect of film hole geometry and blowing ratio on film cooling performance. Applied Thermal Engineering, Vol. 165, pp.114578.

17. Saumweber C., Schulz A. (2012). Free-stream effects on the cooling performance of cylindrical and fan-shaped cooling holes. Journal of Turbomachinery, Vol. 134, No. 6, pp. 061007-1-061007-12.

18. Barigozzi G., Franchini G. Perdichizzi A., Ravelli S. (2010). Film cooling of a contoured end wall nozzle vane through fan-shaped holes. International Journal of Heat and Fluid Flow, Vol. 31, No. 4, pp. 576-585.

19. Hou R., Wen F., Luo Y., Tang X., Wang S. (2019). Large eddy simulation of film cooling flow from round and trenched holes". International Journal of Heat and Mass Transfer, Vol. 144, pp. 118631.

20. Song Y. J., Park S. H., Kang Y. J., Kwak J. S. (2021). Effects of trench configuration on the film cooling effectiveness of a fan-shaped hole. International Journal of Heat and Mass Transfer, Vol. 178, pp. 121655.

21. Stitzel S., Thole K. A. (2004). Flow field computations of combustor-turbine interactions relevant to a gas turbine engine. Journal of Turbomachinery, Vol. 126, No. 1, pp. 122-129.

\section{Biographical notes}

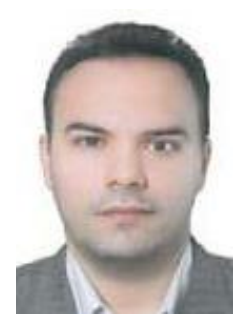

Ehsan Kianpour was born in Iran on 22nd November 1980. He received Master degree (2008) from Malek Ashtar University of Technology and Ph.D degree (2013) from Universiti Teknologi Malaysia. His current interest includes numerical heat transfer, combustion and thermodynamics. Dr Ehsan is an assistant professor at faculty of engineering, najafabad branch, Islamic Azad University, Iran.

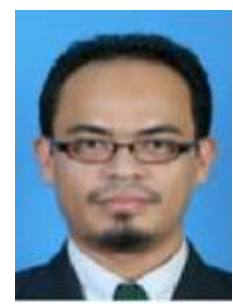

Nor Azwadi C. Sidik was born in Kelantan on $23^{\text {rd }}$ September $1977 . \mathrm{He}$ received Ph.D degree (2014) from Keio University, Japan His current interest includes computational fluid dynamics, numerical methods and fluid structure interaction. $\mathrm{Dr}$ Nor Azwadi is a senior lecturer at Department of Thermofluid, Universiti Teknologi Malaysia, Malaysia. 